Article original

\title{
Digestion des protéines de pois et de soja chez le veau préruminant. I. Taux circulants de nutriments, formation d'anticorps et perméabilité intestinale aux macromolécules
}

\author{
I. Nunes Do Prado 1, R. Toullec ${ }^{1}$, J.P. Lallès ${ }^{1}$, J. Guéguen 2 , \\ J. Hingand ${ }^{3}$ et P. Guilloteau 1
}

avec la collaboration technique de M. Beaufils, S. Boussion, M. Connan, M. Formal, J. Lareynie et Y. Manis

1 INRA, Laboratoire du jeune ruminant, 65, rue de Saint-Brieuc, 35042 Rennes cédex;

2 INRA, Laboratoire de biochimie et technologie des protéines, rue de la Géraudière, 44072 Nantes cédex 03;

3 INRA, Laboratoire de pathologie végétale, 35650 Le Rheu, France

(reçu le 11 novembre 1988, accepté le 6 juin 1989)

\begin{abstract}
Résumé - Trois laits de remplacement (témoin, pois et soja) sont distribués à 6 veaux préruminants. Dans l'aliment témoin, les protéines sont fournies presque exclusivement par de la poudre de lait écrémé. Dans l'aliment pois, 33,5\% d'entre elles sont apportées par une farine de pois dépelliculé prégélatinisée et le reste par de la poudre de lait écrémé. Dans l'aliment soja, elles proviennent pour $73,2 \%$ d'un isolat de soja et pour le reste de la poudre de lactosérum. Au niveau périphérique, les concentrations plasmatiques de triglycérides et sanguines d'azote $\alpha$-aminé libre sont plus faibles avant le repas et plus élevées après, lorsque l'aliment témoin est remplacé par les aliments pois et surtout soja. Cela suggère que l'évacuation gastrique des lipides et celle des protéines sont plus rapides avec ces 2 aliments. Des anticorps se développent contre les protéines de pois mais non contre celles de soja. Les taux plasmatiques de $\beta$-lactoglobuline immunoréactive sont similaires avec les 3 aliments le sixième jour de distribution; la perméabilité intestinale aux macromolécules ne semble donc pas être modifiée, au moins à l'issue de cette période.
\end{abstract}

protéines - digestion — tolérance - veau préruminant

Summary - Digestion of pea and soya-bean proteins in preruminant calves. I. Circulating blood levels of nutrients, antibody formation and gut permeability to macromolecules. Three milk-substitutes (control, pea and soya-bean) were given to 6 preruminant calves. In the control diet protein was almost entirely provided by skim-milk powder. In the pea diet a pregelatinized dehulled pea flour provided $33.5 \%$ of the protein, the remainder being supplied by skim-milk powder. In the soya-bean diet, $73.2 \%$ of the protein were provided by a soya-bean isolate and the remainder by 
whey powder. The concentrations of plasma triglycerides and of free $\alpha$-amino nitrogen in the peripheral blood were lower with the pea and soya-bean diets than with the control diet before the morning meal, but became higher after feeding. That suggested a faster abomasal emptying of fat and protein with the pea and especially the soya-bean diet. Systemic antibody responses were induced against pea protein but not against soya-bean protein. No effect of the diet on the plasma concentration of immunoreactive B-lactoglobulin was apparent after 6 days, suggesting there was no important change in gut permeability at least at that time.

proteins - digestion - tolerance - preruminant calf

\section{INTRODUCTION}

Le remplacement des protéines du lait maternel dans l'alimentation du jeune mammifère est une opération délicate, entraînant fréquemment des réactions d'intolérance. Le veau préruminant constitue à cet égard un modèle d'étude intéressant. Les travaux réalisés jusqu'à présent chez cette espèce montrent que les protéines de substitution provoquent souvent une accélération de l'évacuation gastrique (Guilloteau et al., 1975), sont moins sensibles à l'hydrolyse enzymatique (Jenkins et al., 1980), ont des effets dépressifs sur les sécrétions digestives (Ternouth et al., 1975; Guilloteau et al., 1986a) et conduisent parfois à des troubles de nature allergique (Kilshaw \& Sissons, 1979 a et b).

L'accélération de l'évacuation gastrique est généralement mise en évidence directement, à l'aide de canules duodénales (Guilloteau et al., 1975). Elle peut également être appréciée indirectement, en étudiant l'évolution postprandiale des taux circulants d'acides aminés libres et de triglycérides (Toullec et al., 1979). Cette méthode est moins traumatisante pour l'animal et fournit aussi des informations sur le métabolisme. L'ingestion d'aliments contenant du tourteau de soja cuit peut entraîner des réactions d'hypersensibilité gastro-intestinale se traduisant notamment par des désordres moteurs, des diarrhées, une réduction de la digestibilité (Sissons \& Smith, 1976) et des altérations de la paroi intestinale (Barratt et al., 1978; Seegraber \& Morrill, 1986), entraînant une augmentation de sa perméabilité aux macromolécules telles que la $\beta$-lactoglobuline (Kilshaw \& Slade, 1980). Ces troubles sont probablement de nature allergique et semblent être dus à l'action de la glycinine et de la $\beta$ conglycinine, qui sont les 2 principales globulines du soja. Ils apparaissent après le développement de titres élevés d'lgG et d'lgE dirigées contre ces protéines et ils peuvent être prévenus par la dénaturation de ces dernières (Kilshaw \& Sissons, $1979 a$ et b) et par la distribution d'un médicament anti-allergique (Duvaux et al., 1988). Des troubles d'hypersensitibilité gastro-intestinale ont également été observés avec l'ovalbumine (Kilshaw, 1981) et le gluten de blé (Kilshaw \& Slade, 1982).

Les légumineuses sont les principales sources de protéines de remplacement actuellement disponibles. La digestion des produits issus du pois a été très peu étudiée chez le veau préruminant (Bell et al., 1974). Si de nombreux travaux ont été consacrés aux tourteaux et aux concentrats de soja, il n'en a pas été de même en ce qui concerne les isolats (Pedersen, 1986). Le but de ce travail a été d'étudier l'influence du remplacement des protéines du lait par celles d'une farine de pois 
dépelliculé prégélatinisée ou d'un isolat de soja sur les concentrations circulantes de nutriments, la formation d'anticorps contre les protéines alimentaires et la perméabilité intestinale aux macromolécules. Une publication associée rapportera les résultats concernant les digestibilités iléale et fécale (Nunes do Prado et al., 1989).

\section{MATÉRIEL ET MÉTHODES}

\section{Aliments}

Trois aliments (témoin, pois et soja) ont été comparés (Tableau I). Dans l'aliment témoin, les protéines étaient fournies en quasi-totalité

Tableau I. Composition des aliments et des sources de protéines étudiées.

Constituants

de l'aliment (\%)

Poudre de lait écrémé Spray

Poudre de lactosérum Spray

Pois

Isolat de Soja

Suif 1

Amidon prégélatinisé

Amidon préhydrolysé

Glucose

Chlorure de sodium

Sulfate de magnésium anhydre

Phosphate bicalcique

Chlorure de potassium

L-lysine, $\mathrm{HCl}$

DL-méthionine

Composé minéral vitaminisé
Aliments

Témoin Pois Soja

$\begin{array}{ccc}68,6 & 45,1 & \overline{-} \\ - & - & 44,3 \\ \overline{-} & 32,0 & - \\ 18,8 & \overline{18,4} & 21,0 \\ 2,0 & 1,3 & - \\ 4,0 & - & 6,0 \\ 5,6 & \overline{-} & 6,4 \\ 0,23 & 0,46 & - \\ 0,25 & 0,25 & 0,36 \\ - & 1,35 & -40 \\ - & 0,12 & 0,53 \\ - & 0,15 & 0,35 \\ 0,08 & 0,18 & 0,50\end{array}$

Composition chimique Source de protéines

Aliments

(\% de MS $)^{2}$

Pois $^{3} \quad$ Soja 4

Témoin Pois

Soja

\begin{tabular}{lccccc} 
N x 6,25 & 27,7 & 90,4 & 25,2 & 25,4 & 25,8 \\
Lipides & 2,2 & 4,1 & 19,5 & 19,5 & 19,6 \\
Cendres & 2,7 & 4,0 & 5,8 & 6,5 & 7,2 \\
Calcium & 0,14 & 0,13 & 0,96 & 0,95 & 0,97 \\
Phosphore & 0,42 & 0,77 & 0,72 & 0,84 & 0,89 \\
Magnésium & 0,10 & 0,04 & 0,15 & 0,15 & 0,15 \\
Sodium & 0,07 & 0,40 & 0,46 & 0,45 & 0,45 \\
Potassium & 0,87 & 0,27 & 1,21 & 1,10 & 1,11 \\
Extractif non azoté & 67,4 & 1,5 & 49,5 & 48,6 & 47,5 \\
\hline
\end{tabular}

1 Homogénéisé dans du lait écrémé (témoin et pois) ou du lactosérum (soja) concentrés avant séchage par le procédé Spray (35\% de lipides/MS); ${ }^{2}$ MS : matière sèche; ${ }^{3}$ Coopérative de traitement des produits de la pêche, BP 239, 62203 Boulogne-sur-Mer; 4 Purina protein 610, Duquesne Purina, 2028 X, 76040 Rouen Cédex; 3,4 Activité antitrypsique : 1,1 et 3,7 unités de trypsine inhibée/mg de MS, respectivement, dans le pois et le soja. 
par de la poudre de lait écrémé. Dans l'aliment pois, elles provenaient pour $33,5 \%$ d'une farine de pois dépelliculé prégélatinisée, le reste étant apporté par de la poudre de lait écrémé $(65,7 \%)$ et des acides aminés de synthèse $(0,8 \%)$. Enfin, dans l'aliment soja, les protéines étaient fournies par un isolat de soja $(73,2 \%)$, de la poudre de lactosérum $(24 \%)$ et des acides aminés de synthèse $(2,8 \%)$. Les aliments pois et soja ont été obtenus en mélangeant, au moment de l'emploi, la source de protéines étudiées et un aliment complémentaire apportant tous les autres ingrédients. La proportion de farine de pois a été choisie de manière à ne pas apporter un taux excessif d'amidon. En revanche, l'isolat de soja a apporté une plus grande part des protéines parce qu'il était dépourvu d'amidon. La farine de pois et l'isolat de soja avaient des activités antitrypsiques voisines par rapport aux protéines (4 unités de trypsine inhibée/mg) et équivalentes à environ $4 \%$ de celle trouvée dans le soja cru (Rackis, 1974). Les autres protéines semblaient également avoir été largement dénaturées au cours de la préparation des produits. Ainsi, la légumine immunoréactive ne représentait plus qu'environ $1 \%$ des protéines totales du pois prégélatinisé, alors qu'elle atteint couramment $20 \%$ dans le pois cru (Guéguen \& Barbot, 1988). La glycinine et la $\beta$-conglycinine n'étaient plus détectables sous forme immunoréactive dans l'isolat de soja.

\section{Animaux, régimes et prélèvements}

Six veaux mâles préruminants de race Holstein, achetés à l'âge d'environ $8 \mathrm{j}$, ont été munis d'une canule réentrante dont la partie proximale a été posée à la fin de liléon et la partie distale dans le caecum, selon les modalités décrites par Guilloteau et al. (1986b). Les animaux étaient âgés de $63 \pm 2 \mathrm{j}$ (moyenne \pm écart type de la moyenne) et pesaient $96 \pm 3 \mathrm{~kg}$ au moment de l'opération. Ils ont été nourris exclusivement avec un lait de remplacement dont les protéines étaient en totalité d'origine laitière jusqu'au début de l'essai. Chaque veau a reçu chacun des 3 régimes expérimentaux (témoin, pois et soja) dans un ordre différent, au cours de 3 périodes successives de 3 semaines, dont la première a commencé 1 à 2 semaines après l'opération. Les changements d'aliment ont été effectués en $4 \mathrm{j}$, en introduisant $25,50,75$ et $100 \%$ d'aliment à étudier dans la ration le premier, le deuxième, le troisième et le quatrième jour de chaque période, respectivement. Les veaux ont été alimentés au seau, 2 fois par jour (à $8 \mathrm{~h} 30$

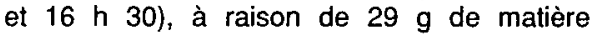
sèche/kg de poids vif $0,75 /$ repas.

Des prises de sang ont été effectuées dans une veine jugulaire externe $6 \mathrm{~h}$ après le repas du matin, la veille du début de la première période, ainsi qu'avant et $1,2,3$ et $4 \mathrm{~h}$ après le repas du matin, au cours du septième jour de chaque période. Environ $22 \mathrm{ml}$ de sang ont été répartis entre 2 tubes renfermant $0,1 \mathrm{ml}$ de solution d'héparine $(5000 \mathrm{Ul} / \mathrm{ml})$. Un $\mathrm{ml}$ de sang a été immédiatement transféré dans une solution d'acide trichloracétique, de manière à obtenir une concentration finale de $2,25 \%$ pour l'acide. Le reste du sang a été centrifugé et le plasma prélevé et conservé à $-18^{\circ} \mathrm{C}$ jusqu'aux analyses.

\section{Analyses et calculs}

L'activité antitrypsique a été mesurée dans le pois et le soja par la méthode de I'AOAC (1983). Les teneurs plasmatiques en glucose et triglycérides, ainsi que le taux d'azote $\alpha$-aminé libre dans le sang total ont été mesurés selon des techniques décrites antérieurement (Toullec et al., 1979). Les titres d'anticorps plasmatiques dirigés contre les extraits totaux de protéines de pois et de soja, ainsi que contre les deux principales globulines de pois (légumine et viciline) et de soja (glycinine et Bconglycinine), ont été déterminés par hémagglutination passive (Herbert, 1973) et par ELISA (Heppell et al., 1987), afin de comparer les 2 méthodes. Les extraits totaux ont été obtenus à l'aide de tampon phosphate à $\mathrm{pH}$ 7,4 , la légumine et la viciline par la méthode de Guéguen et al. (1984) et la glycinine et la Bconglycinine selon les techniques de Thanh \& Shibasaki (1976) et de King (1977), respectivement. Pour le titrage des anticorps par ELISA, les antigènes ont été adsorbés sur des plaques de polystyrène Nunc (référence 469 914) et la détection des anticorps fixés par les 
antigènes a été effectuée à l'aide d'lgG de lapin anti-lgG de bovins conjuguées à la phosphatase alcaline (Sigma, référence $\mathrm{A}$ 7914); en fait, cette préparation n'étant pas spécifique des chaînes $\gamma$, elle reconnaît également les IgA et les IgM. Les titres d'anticorps sont les nombres maximaux de dilutions au demi permettant d'obtenir l'agglutination des érythrocytes de mouton enrobés d'antigènes ou une densité optique de 0,2 en ELISA, en partant d'une dilution initiale du plasma au vingtième.

La légumine immunoréactive a été dosée dans le plasma par une technique ELISA en "sandwich indirect", en utilisant 2 immunsérums antilégumine préparés respectivement chez le mouton et le lapin. Les sérums ont été dessalés par tamisage moléculaire (Ultrogel ACA 202 (BF), puis les immunoglobulines ont été séparées des albumines par chromatographie sur résine échangeuse d'ions (DEAETrisacryl M IBF), en utilisant un tampon de $\mathrm{pH}$ 8,8 contenant par litre 25 mmoles de TRIS, 35 mmoles de chlorure de sodium et $1 \mathrm{ml}$ d'acide chlorhydrique $12 \mathrm{M}$.

Pour le dosage de la légumine, chaque puits a reçu successivement $200 \mu \mathrm{l}$ :

- de solution d'immunoglobulines de brebis $(5 \mu \mathrm{g} / \mathrm{ml}$ de tampon bicarbonate à $\mathrm{pH} 9,7)$;

- d'échantillon à doser ou de gamme. Les plasmas ont été dilués au demi ou au quart dans du PBST (tampon phosphate à pH 7,4 contenant $0,5 \mathrm{ml}$ de Tween 20 par litre). La gamme a été préparée dans du PBST et diluée dans du plasma de bœuf, de manière à obtenir les mêmes proportions de plasma et de PBST que dans les échantillons à doser;

- de solution d'immunoglobulines de lapin (5 $\mu \mathrm{g} / \mathrm{ml}$ de PBST);

- de solution d'lgG de chèvre anti-lgG de lapin conjuguées à la phosphatase alcaline (Sigma, référence $A 8025$, dilution $1 / 3000$ dans du PBST);

- de solution de substrat de la phosphatase (1 $\mathrm{mg}$ de nitro-phényl-phosphate de sodium hexahydraté par litre de tampon Boehringer, référence 726 915).
Les immunoglobulines, les échantillons et la gamme ont été laissés dans les puits pendant $3 \mathrm{~h}$ à $37^{\circ} \mathrm{C}$ ou $15 \mathrm{~h}$ à $4^{\circ} \mathrm{C}$ et les conjugués pendant $2 \mathrm{~h}$ à $37^{\circ} \mathrm{C}$. Les puits ont été rincés 4 fois au PBST après élimination de chacun de ces produits. La densité optique a été lue à $405 \mathrm{~nm}$ au bout d'environ $30 \mathrm{~min}$. La gamme a été linéraire entre 10 à $120 \mathrm{ng}$ de légumine par $\mathrm{ml}$ de plasma, dans le cas d'une dilution au quart dans le PBST.

La $\beta$-lactoglobuline immunoréactive a été dosée dans le plasma à l'aide d'une technique ELISA par compétition en utilisant un immunsérum préparé chez le cobaye. La $\beta$-lactoglobuline pure était un don de J.P. Pélissier, du laboratoire de biochimie et technologie laitières de I'INRA (78350 Jouy-en-Josas). Les échantillons de plasma à doser ont été mis en contact avec l'immunsérum, dans des tubes de verre maintenus à $37^{\circ} \mathrm{C}$ pendant $3 \mathrm{~h}$ et agités toutes les $30 \mathrm{~min}(500 \mu \mathrm{l}$ de plasma, $500 \mu \mathrm{lde}$ PBST et $1000 \mu$ l d'immunsérum préalablement dilué 10000 fois dans du PBST). La gamme a été traitée de la même façon, sauf qu'elle a été préparée dans du PBST et mélangée à du plasma de bceuf et à l'immunsérum dilué (respectivement 500,500 et $1000 \mu \mathrm{l}$ ). Chaque puits de dosage a reçu successivement la solution de $\beta$-lactoglobuline $(5 \mu \mathrm{l} / \mathrm{ml}$ de tampon carbonate), l'échantillon ou la gamme mis en contact avec l'immunsérum comme indiqué cidessus et des IgG de chèvre anti-lgG de cobaye conjuguées à la phosphatase alcaline (Sigma, référence A7664). Les autres détails du dosage ont été identiques à ceux employés pour la légumine. La gamme a été linéaire entre 20 et $1000 \mathrm{ng} / \mathrm{ml}$ de plasma.

La glycinine et la $\beta$-conglycinine immunoréactives ont été recherchées dans l'isolat de soja à l'aide d'une technique ELISA semblable à celle utilisée pour la $\beta$-lactoglobuline, à l'aide d'antisérums préparés chez le lapin.

Les différences observées en fonction du temps ont été testées par la méthode des couples (test $t$ de Student). Les effets du régime ont été soumis à une analyse de variance et les moyennes classées à l'aide du test de Newman \& Keuls. 


\section{RÉSULTATS}

\section{Azote $\alpha$-aminé libre}

Avec l'aliment témoin, la teneur sanguine en azote $\alpha$-aminé libre a tendance à baisser après le repas, mais cette évolution n'est pas significative (Fig. 1). En revanche, avec les 2 autres aliments, la teneur augmente significativement après le repas, plus rapidement dans le cas du soja que dans celui du pois. Elle est plus faible qu'avec l'aliment témoin avant le repas, mais plus élevée $2 \mathrm{~h}$ après pour l'aliment soja et $4 \mathrm{~h}$ après pour les aliments pois et soja. Enfin, elle est plus élevée avec l'aliment soja qu'avec l'aliment pois $2 \mathrm{~h}$ après le repas.

\section{Triglycérides}

Avec l'aliment témoin, la teneur en triglycérides du plasma baisse jusqu'à $3 \mathrm{~h}$ après la prise du repas (Fig. 1); bien qu'elle remonte entre 3 et $4 \mathrm{~h}$, elle demeure toujours significativement plus faible qu'à jeun. Avec les 2 autres aliments, elle augmente pendant les 2 premières heures postprandiales, surtout dans le cas du soja; elle baisse entre 3 et $4 \mathrm{~h}$ avec l'aliment soja, mais pas avec l'aliment pois. Elle est plus faible qu'avec l'aliment témoin avant le repas mais plus élevée à partir de la première heure postprandiale dans le cas du soja et à partir de la seconde dans le cas du pois. Elle est plus élevée avec l'aliment soja qu'avec l'aliment pois de la première à la troisième heure postprandiale.
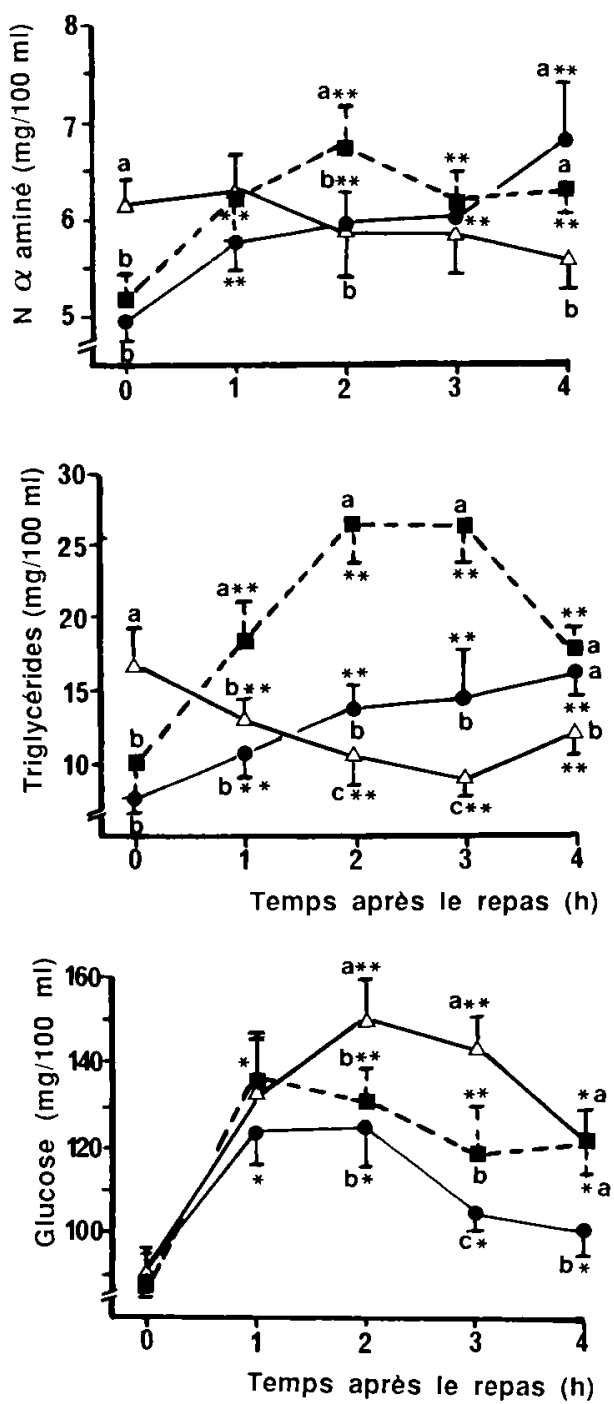

Fig. 1. Evolution postprandiale de la teneur sanguine en azote $\alpha$-aminé libre et des teneurs plasmatiques en triglycérides et en glucose avec les aliments témoin $(\Delta)$, pois $(0)$ et soja $(\square) ;{ }^{* *},{ }^{\star}$ : différence significative par rapport à la valeur observée à jeun $(P<0,01$ et 0,05 , respectivement); $a, b, c$ : à un temps donné, les valeurs accompagnées de lettres différentes sont significativement différentes $(P<$ $0,05)$. 


\section{Glucose}

La teneur plasmatique en glucose est similaire avant le repas avec les trois aliments et augmente au cours de la première heure postprandiale (Fig. 1). Avec l'aliment témoin, elle continue à croitre pendant la seconde heure, puis elle diminue. Avec les 2 autres aliments, elle est moins élevée qu'avec l'aliment témoin à l'issue de la deuxième et de la troisième heure, ainsi qu'à la fin de la quatrième dans le cas du pois. Enfin, elle est plus forte avec l'aliment soja qu'avec l'aliment pois 3 et $4 \mathrm{~h}$ après le repas.

\section{Anticorps}

En hémagglutination passive, les titres d'anticorps dirigés contre l'extrait total de protéines de pois, la légumine et la viciline sont nuls avant le début de la distribution de l'aliment pois. II en est de même le septième jour, après 12 repas d'aliment pois. En revanche, $21 \mathrm{j}$ plus tard, c'est à dire $7 \mathrm{j}$ après la fin de la distribution d'aliment pois, les titres s'élèvent contre l'extrait total $(3,5 \pm 0,5 ; P<0,01$ par rapport à la valeur initiale) et surtout contre la légumine $(5,9 \pm 0,3 ; P<0,01)$; bien que devenant positifs, ils demeurent très faibles contre la viciline $(1,0 \pm 0,4$; $P<0,05)$. En ELISA, les titres observés avant le début de la distribution d'aliment pois sont positifs et très voisins contre les 3 catégories d'antigènes (en moyenne 5,6 $\pm 0,2$ ). Comme en hémagglutination passive, ils ne changent pas pendant les 7 premiers jours mais sont plus élevés $(P<0,01) 21 \mathrm{j}$ plus tard $(10,3 \pm 2,0 ; 10,4$ $\pm 0,4$ et $9,4 \pm 1,6$ respectivement contre l'extrait total, la légumine et la viciline).

En hémagglutination passive, les anticorps antisoja ne sont pas non plus détectés avant la distribution d'aliment soja. De faibles titres sont observés le septième jour contre la glycinine $(1,0 \pm$ $0,6 ; P<0,05)$ et 21 j plus tard contre la glycinine et l'extrait total $(1,0 \pm 0,8$ et $1,0 \pm$ $0,5 ; P<0,05)$. En revanche, aucune activité ne peut être mise en évidence contre la $\beta$-conglycinine. En ELISA, les titres observés avant le début de la distribution d'aliment soja sont positifs contre l'extrait total $(6,2 \pm 0,7)$, la glycinine $(4,7 \pm 0,3)$ et la $\beta$-conglycinine $(4,7 \pm 0,3)$. Les titres trouvés le septième jour $(6,5 \pm$ 0,$3 ; 5,9 \pm 0,6$ et $6,5 \pm 0,6$ ) et 21 j plus tard $(7,0 \pm 0,0 ; 6,0 \pm 0,0$ et $6,7 \pm 0,3)$ ne sont pas significativement plus élevés.

\section{$\beta$-lactoglobuline et légumine}

Aucune différence n'est observée selon le régime ou le temps après le repas dans le taux plasmatique de $\beta$-lactoglobuline immunoréactive qui est compris entre 120 et $230 \mathrm{ng} / \mathrm{ml}$. La légumine immunoréactive ne peut être détectée à aucun moment dans le plasma, le septième jour de distribution d'aliment pois ou $21 \mathrm{j}$ plus tard.

\section{DISCUSSION}

\section{Concentrations circulantes de nutri- ments}

Les évolutions postprandiales observées pour les teneurs plasmatiques en glucose et en triglycérides, ainsi que pour la teneur sanguine en azote $\alpha$-aminé libre, avec l'aliment témoin, sont en accord 
avec celles obtenues par différents auteurs avec le lait entier ou les laits de remplacement riches en poudre de lait écrémé (Toullec et al., 1979; Beynen \& Van Gils, 1983). L'augmentation rapide du taux de glucose, par rapport à ceux de triglycérides et d'azote $\alpha$-aminé libre, reflète l'évacuation gastrique beaucoup plus rapide du lactose, par comparaison avec celles des lipides et des protéines. Malgré l'accroissement des quantités de lipides et de protéines arrivant dans le duodénum, les taux circulants des produits résultant de leur digestion stagnent ou baissent parce que l'élévation de l'insulinémie favorise leur prélèvement par les tissus et réduit la protéolyse musculaire.

Les valeurs plus faibles observées avant le repas pour les taux circulants de triglycérides et d'azote $\alpha$-aminé libre, durant les périodes de distribution des aliments pois et soja, indiquent que la caillette est alors probablement plus vide que lors de la période de distribution de l'aliment témoin. Les augmentations postprandiales considérables entraînées par l'ingestion de l'aliment soja sont dues à l'absorption de quantités massives de lipides et d'acides aminés, résultant de l'évacuation gastrique plus rapide des lipides et des protéines, en l'absence de coagulation. Elles ont également été observées avec d'autres aliments incoagulables (Toullec et al., 1979; Beynen \& Van Gils, 1983; Petit et al., 1988). La diminution plus rapide du taux plasmatique de glucose pourrait résulter d'une augmentation de la sécrétion d'insuline ou de la sensibilité des tissus à cette hormone (Grizard et al., 1982). Cependant, cette augmentation ne semble pas suffire à empêcher l'accroissement des taux circulants de triglycérides et d'azote $\alpha$-aminé libre. Le prélèvement des acides aminés par les tissus pourrait également être limité par l'équilibre moins satisfaisant des protéines du soja par rapport à celles du lait (Guilloteau et al., 1981).

Avec l'aliment pois, les modifications entraînées dans l'évolution postprandiale des taux circulants de triglycérides et d'azote $\alpha$-aminé sont moindres qu'avec l'aliment soja. Cela indique que l'évacuation gastrique des lipides et des protéines est moins accélérée dans le cas de l'aliment pois. Cela n'est pas surprenant car, dans cet aliment, une part importante des protéines est apportée par du lait écrémé. Cependant, il est probable que le coagulum formé est moins ferme et qu'il se désagrège plus rapidement que dans le cas de l'aliment témoin, comme en témoigne l'accroissement des taux circulants de triglycérides et d'azote $\alpha$ aminé. Quoi qu'il en soit, ni l'aliment pois ni l'aliment soja n'entraînent de blocage de l'évacuation gastrique au cours des premières heures postprandiales, comme cela a été observé chez le veau recevant un aliment riche en tourteau de soja cuit (Sissons \& Smith, 1976). Avec l'aliment pois, le taux de glucose augmente moins après le repas qu'avec l'aliment témoin et redescend ensuite plus vite qu'avec l'aliment soja. Cela pourrait résulter de la teneur plus élevée en amidon de l'aliment pois, dont la digestion est plus lente que celle du lactose et a lieu en partie dans le gros intestin (Nunes do Prado et al., 1989), en y fournissant des acides gras volatils et de l'acide lactique, au lieu du glucose (Assan \& Thivend, 1976).

\section{Aspects antigéniques}

Les titres d'anticorps trouvés par hémagglutination passive avant le début de la période expérimentale sont nuls à la fois contre les protéines de pois et contre 
celles de soja. Les résultats concernant le soja ne s'accordent pas avec ceux obtenus, en utilisant la même technique, par Guilloteau et al., (1986a) et Pedersen (1986). Les anticorps antisoja détectés par ces auteurs dans le plasma de veaux n'ayant pas consommé de soja ont été considérés comme pouvant être d'origine colostrale. Ainsi, Pedersen (1986) a observé la présence d'anticorps ayant une activité antisoja dans les colostrums de vaches recevant du tourteau de soja, dans leurs plasmas et dans les plasmas des veaux ayant ingéré ces colostrums; cependant, le titre d'anticorps diminuait après la période colostrale, chez les veaux ne recevant pas d'aliment contenant du soja. En revanche, par hémagglutination passive, nous n'avons pas détecté d'activité contre les protéines de soja ou celles de pois dans un colostrum de mélange ou dans les immunoglobulines extraites de ce colostrum, ainsi que dans les plasmas sanguins obtenus $26 \mathrm{~h}$ après la naissance chez 12 veaux ayant reçu 3 repas de l'un de ces 2 produits (Nunes do Prado \& Toullec, résultats non publiés). Quoi qu'il en soit, dans la présente expérience, les veaux ont environ $80 \mathrm{j}$ au début des mesures et il est vraisemblable que peu d'anticorps d'origine colostrale subsistent encore (Ischikawa \& Konishi, 1982). Les titres initiaux de 5 à 6 trouvés par ELISA contre tous les antigènes étudiés sont probablement dus en majeure partie à l'adsorption non spécifique des immunoglobulines en solution peu diluée. En effet, nous avons obtenu des valeurs très voisines par cette technique chez les veaux de $26 \mathrm{~h}$ ayant reçu du colostrum et évoqués ci-dessus. II en a été de même pour Heppell et al. (1987), en mesurant par ELISA le titre anti-soja, chez des veaux de 13 semaines nourris avec un aliment dont les protéines provenaient exclusivement du lactosérum; en revanche, ce titre était de 12 avec un aliment contenant du tourteau de soja cuit. Les variations des titres d'anticorps avec le temps sont similaires avec les 2 méthodes que nous avons utilisées, sauf contre la viciline : dans ce cas, l'ELISA se montre plus sensible.

La distribution de l'aliment pois entraine la formation d'anticorps dirigés contre les protéines de pois. Cependant, ce phénomène n'est pas encore détectable après 1 semaine de régime, ce qui est conforme à l'évolution observée dans le temps par Pedersen (1986) et Heppell et al. (1987), avec le régime soja évoqué au paragraphe précédent. Le fait qu'en hémagglutination passive un titre plus élevé soit obtenu contre la légumine que contre la viciline ne signifie pas obligatoirement que la première soit plus immunogène que la seconde. En effet, la technique utilisée ne permet pas d'estimer les valeurs absolues des concentrations d'anticorps, à cause des différences dans l'avidité des anticorps et dans la quantité d'anticorps complexés avec les antigènes (Pedersen, 1986). Les augmentations de titres que nous obtenons entre le début et la fin de l'essai sont moindres que celles observées en 4 semaines par Pedersen (1986) et Heppell et al. (1987) chez des veaux recevant un aliment contenant du tourteau de soja cuit. On pourrait en conclure que les protéines de pois prégélatinisé sont moins immunogènes que celles du tourteau de soja d'autant plus que ses protéines semblent avoir été largement dénaturées par le traitement; cependant, les mêmes réserves que celles décrites ci-dessus à propos des écarts entre les titres antilégumine et antiviciline, empêchent d'être affirmatif sur ce point, d'autant plus qu'il s'agit de comparer des résultats de laboratoires différents. Par ailleurs, les très faibles variations des titres antisoja dans le plasma des veaux sont en accord avec l'absence apparente de glycinine et de B- 
conglycinine immunoréactives dans l'isolat. $\mathrm{Ce}$ dernier est donc pratiquement dépourvu d'activité antigénique contrairement au tourteau de soja et, à cet égard, il se comporte comme un concentrat traité à l'alcool (Kilshaw \& Sissons, 1979a et b).

La formation d'anticorps est une réponse à l'absorption de petites quantités d'antigènes par la paroi intestinale (Walker \& Isselbacher, 1974). Cependant, la légumine immunoréactive n'est pas détectée dans le plasma des veaux à la fin de la première semaine de distribution d'aliment pois. Walker et al. (1975) ont montré que l'absorption d'antigène ne peut être mise en évidence chez le rat qu'au cours des premiers jours d'ingestion; de même, en distribuant à des veaux un lait de remplacement contenant de la farine de pois cru, nous n'avons observé la présence de légumine immunoréactive qu'après le deuxième et le troisième repas (Nunes do Prado et al., 1988). II se pourrait que la synthèse d'anticorps, bien que non décelable avec les méthodes employées, soit suffisante pendant la première semaine pour saturer la totalité des épitopes de la légumine absorbée le septième jour, empêchant ainsi sa détection par ELISA.

La $\beta$-lactoglobuline est considérée comme ne provoquant pas la formation d'anticorps chez le veau et les variations de son taux circulant peuvent donc être utilisées pour caractériser la perméabilité intestinale aux macromolécules qui augmente en cas d'altération de la paroi (Kilshaw \& Slade, 1980). La majeure partie de l'immunoréactivité normalement présente dans le plasma du veau nourri au lait est due à des immun-complexes d'origine colostrale dans lesquels la Blactoglobuline n'est pas entièrement saturée par les anticorps (Kilshaw \& Slade, 1981). Dans notre essai, la teneur plasmatique en $\beta$-lactoglobuline immunoréactive n'évolue significativement ni avec le régime ni avec le temps écoulé depuis le repas. Cela indique qu'au moment des mesures ni l'aliment pois ni l'aliment soja n'avaient entraîné d'altération de la paroi intestinale décelable par la méthode utilisée. En distribuant à des veaux un aliment contenant de la farine de pois cru, nous avons observé une augmentation de la teneur plasmatique en $\beta$-lactoglobuline entre la troisième et la septième semaine de régime (Nunes do Prado et al., 1988).

La mise en évidence d'éventuelles réactions d'hypersensitibilité gastro-intestinale aurait donc nécessité une durée expérimentale plus longue que celle utilisée dans le présent essai. Dans le cas de l'isolat de soja, il ne doit cependant pas se produire d'augmentation de la perméabilité intestinale aux macromolécules, compte tenu de son absence d'activité antigénique.

En conclusion, le remplacement des protéines du lait par celles d'une farine de pois prégélatinisée ou d'un isolat de soja entraîne une accélération de l'absorption des lipides et des acides aminés, qui témoigne d'une augmentation de la vitesse d'évacuation gastrique. Les veaux forment des anticorps contre les protéines de pois mais très peu contre celles du soja. Cependant, aucun signe clinique d'intolérance n'apparaît à l'égard du pois dans les conditions de l'essai.

\section{REMERCIEMENTS}

A Jeannine Quillet pour avoir réuni les données bibliographiques, à J.P. Pélissier pour le don de $\beta$-lactoglobuline pure, à la société Sanders pour le dosage des facteurs antitrypsiques, ainsi qu'à la coopérative de traitement des produits de la pêche et à Duquesne Purina qui ont gracieusement fourni la farine de pois et l'isolat de soja. 


\section{RÉFÉRENCES}

AOAC (1983) Official Methods of Analysis, BA 12-75. Association of Official Agricultural Chemists, Washington, D.C.

Assan B.E. \& Thivend P. (1976) Intestinal digestion of tropical starches by the preruminant calf. Proc. Nutr. Soc. 35, 104A105A

Barratt M.E.J., Strachan P.J. \& Porter P. (1978) Antibody mechanisms implicated in digestive disturbances following ingestion of soya protein in calves and piglets. Clin. Exp. Immunol. 31, 305-312

Bell J.M., Royan G.F. \& Youngs C.G. (1974) Digestibility of pea protein concentrate and enzyme-treated pea flour in milk replacers for calves. Can. J. Anim. Sci. 54, 355-362

Beynen A.C. \& Van Gils L.G.M. (1983) Postprandial changes in the levels of lipids, glucose, urea and nonprotein nitrogen in the serum of veal calves fed milk replacers containing either milk powder or soybean protein concentrate. Z. Tierphysiol. Tierernähr.

Futtermittelkd. 49, 49-56

Duvaux C., Sissons J.W., Heppell L., Toullec R. \& Guilloteau P. (1988) Mécanismes impliqués dans la réaction allergique intestinale aux protéines de soja chez le veau préruminant. Reprod. Nutr. Dév. 28, suppl. 1, 159-160

Grizard J., Toullec R., Guilloteau P. \& PatureauMirand P. (1982) Influence de la cinétique d'évacuation gastrique de l'aliment sur l'insulinémie chez le veau préruminant. Reprod. Nutr. Dév. 22, 475-484

Guéguen J. \& Barbot J. (1988) Quantitative and qualitative variability of pea (Pisum Sativum L.) protein composition. J. Sci. Food Agric. 42, 209-224

Guéguen J., Vu A.T. \& Schaeffer F. (1984) Large-scale purification and characterization of pea globulins. J. Sci. Food Agric. 35, 10241033

Guilloteau P., Corring T., Chayvialle J.A., Bernard C., Sissons J.W. \& Toullec R. (1986a) Effect of soya protein on digestive enzymes, gut hormone and anti-soya antibody plasma levels in the preruminant calf. Reprod. Nutr. Dev. 26, 717-728

Guilloteau P., Paruelle J.L., Toullec R. \& Mathieu C.M. (1975) Utilisation des protéines par le veau préruminant à l'engrais. III. Influence du remplacement des protéines du lait par celles du poisson sur la vidange stomacale. Ann. Zootech. 24, 243-253

Guilloteau P., Toullec R., Grongnet J.F., Patureau-Mirand P., Prugnaud J. \& Sauvant D. (1986b) Digestion of milk, fish and soya-bean protein in the preruminant calf : flow of digesta, apparent digestibility at the end of the ileum and amino acid composition of ileal digesta. Br. J. Nutr. 55, 571-592

Guilloteau P., Toullec R., Patureau-Mirand P. \& Prugnaud J. (1981) Importance of the abomasum in the digestion in preruminant calf. Reprod. Nutr. Dev. 21, 885-899

Heppell L.M.J., Sissons J.W., Stobo I.J.F., Thurston S.M. \& Duvaux C. (1987) Immunological intolerance in calves fed with antigenic soyabean protein. In : Food Allergy (Chandra R.K., ed.) Nutrition Research Education Foundation, St John's, Newfoundland

Herbert W.J. (1973) Passive haemagglutination with special reference to the tanned cell technique. In : Handbook of Experimental Immunology. (Weir D.M., ed.) vol. 1, Blackwell Scientific Publications, Oxford

Ishikawa H. \& Konishi T. (1982) Changes in serum immunoglobulin concentrations of young calves. Jpn. J. Vet. Sci. 44, 555-563

Jenkins K.J., Mahadevan S. \& Emmons D.B. (1980) Susceptibility of proteins used in calf milk replacers to hydrolysis by various proteolytic enzymes. Can. J. Anim. Sci. 60, 907-914

Kilshaw P.J. (1981) Gastrointestinal hypersensitivity in the preruminant calf. In : Current Topics in Veterinary Medicine and Animal Science. (Bourne F.J., ed.) vol. 12, The Mucosal Immune System. Martinus Nijhoff Publishers, The Hague

Kilshaw P.J. \& Sissons J.W. (1979a) Gastrointestinal allergy to soyabean protein in preruminant calves. Antibody production and digestive disturbances in calves fed heated soyabean flour. Res. Vet. Sci. 27, 361-365

Kilshaw P.J. \& Sissons J.W. (1979b) Gastrointestinal allergy to soyabean protein in preruminant calves. Allergenic constituents of soyabean products. Res. Vet. Sci. 27, 366-371

Kilshaw P.J. \& Slade H. (1980) Passage of ingested protein into the blood during gastrointestinal hypersensitivity reactions : experiments 
in the preruminant calf. Clin. Exp. Immunol. 41, 572-582

Kilshaw P.J. \& Slade H. (1981) Milk proteins immune complexes in the cow and calf. $J$. Reprod. Immunol. 3, 227-236

Kilshaw P.J. \& Slade H. (1982) Villus atrophy and crypt elongation in the small intestine of preruminant calves fed with heated soyabean flour or wheat gluten. Res. Vet. Sci. 33, 305308

King N.L. (1977) Heat-induced complex formation between myosin and soyabean $7 \mathrm{~S}$ globulin. J. Agric. Food Chem. 25, 166-171

Nunes do Prado I., Toullec R., Guilloteau P. \& Guéguen J. (1989) Digestion des protéines de pois et de soja chez le veau préruminant. II. Digestibilité apparente à la fin de l'iléon et du tube digestif. Reprod. Nutr. Dev. 29, sous presse

Nunes do Prado I., Toullec R., Lallès J.P., Hingand L. \& Guéguen J. (1988) Anticorps contre les protéines alimentaires et perméabilité intestinale aux macromolécules chez le veau préruminant recevant de la farine de pois cru. Reprod. Nutr. Dev. 28, suppl. 1, 157-158

Pedersen H.E. (1986) Studies of soyabean protein intolerance in the preruminant calf. $\mathrm{Ph}$. D. Thesis University of Reading, Angleterre

Petit H.V., Ivan M. \& Brisson G.J. (1988) Digestibility and blood parameters in preruminant calves fed a clotting or a nonclotting milk replacer. J. Anim. Sci. 66, 986-991

Rackis J.J. (1974) Biological and physiological factors in soybeans. J. Am. Oil. Chem. Sci. 51, 161A-174A
Seegraber F.J. \& Morrill J.L. (1986) Effect of protein source in calf milk replacers on morphology and absorptive ability of small intestine. J. Dairy Sci. 69, 460-469

Sissons J.W. \& Smith R.H. (1976) The effects of different diets, including those containing soya products on digesta movement and water and nitrogen absorption in the small intestine of the preruminant calf. Br. J. Nutr. 36, 421-438

Ternouth J.H., Roy J.H.B., Thompson S.Y., Toothill J., Gillies C.M. \& Edwards-Webb J.D. (1975) Concurrent studies of the flow of digesta in the duodenum and of exocrine pancreatic secretion of calves. 3 - Further studies on the addition of fat to skim milk and the use of nonmilk proteins in milk substitute diets. $B r$. J. Nutr. 33, 181-196

Thanh V.H. \& Shibasaki K. (1976) Major protein of soya seeds. A straightforward fractionation and their characterization. J. Agric. Food Chem. 24, 1 117-1 121

Toullec R., Guilloteau P. \& Coroller J.Y. (1979) Influence de la cinétique d'évacuation gastrique de l'aliment sur l'absorption chez le veau préruminant. Ann. Biol. Anim. Bioch. Biophys. 19, 729-732

Walker W.A. \& Isselbacher K.J. (1974) Uptake and transport of macromolecules by the intestine. Possible role in clinical disorders. Gastroenterology 67, 531-550

Walker W.A., Wu M., Isselbacher K.J. \& Bloch K.J. (1975) Intestinal uptake of macromolecules. III - Studies on the mechanism by which immunization interferes with antigen uptake. J. Immunol. 115, 854-861 